\title{
Evaluation of Bedside Delivery of Medications Before Discharge: Effect on 30-Day Readmission
}

\author{
Jodi B. Segal, MD, MPH; Ariella Apfel, MHS; Daniel J. Brotman, MD; \\ Kenneth M. Shermock, PharmD, PhD; and Jeanne M. Clark, MD, MPH
}

\begin{abstract}
BACKGROUND: This study is an evaluation of a discharge intervention that occurred in multiple hospitals across Maryland. In this program, patients received medications at their bedside before discharge with the goal of reducing the risk of primary nonadherence to prescribed medications.

OBJECTIVE: To test if the intervention reduced the risk of 30-day readmission for the patients who received bedside medication delivery relative to comparable patients who did not receive bedside medication delivery.

METHODS: This was a retrospective cohort study. Patients who received the intervention were linked to their claims data in the Maryland Health Information Exchange. These patients were matched on age, sex, diagnosis-related group, and hospital to a set of patients who did not receive the intervention. We used propensity score matching, as well as inverseprobability weighting, to account for residual differences between the treated and comparison patients. With robust Poisson regression, adjusting for hospital, we generated risk ratios for 30 -day readmission and explored risk ratios in key subgroups.
\end{abstract}

RESULTS: The cohort included 6,167 inpatients who received medications at bedside and 28,546 who did not from 10 Maryland hospitals. They were $60 \%$ female, $61 \%$ white, and $31 \%$ African American; the average age was 56 years. The risk ratio for readmission, comparing the intervention group to the propensity score-matched comparison group, was 1.21 (95\% $\mathrm{Cl}=0.96-1.5)$. Inverse-probability weighting yielded a similar result (1.19 [95\% $\mathrm{Cl}=0.98-1.45])$.

CONCLUSIONS: In this study, the isolated intervention of bedside medication delivery did not reduce 30 -day readmission risk. We expect it may have favorable outcomes on other metrics such as primary nonadherence and patient satisfaction. It may also have a favorable effect when bundled with other care transition activities. As an isolated intervention, however, bedside medication delivery is unlikely to affect 30 -day readmission rates.

J Manag Care Spec Pharm. 2020;26(3):296-304

Copyright @ 2020, Academy of Managed Care Pharmacy. All rights reserved.

\section{What is already known about this subject}

Bedside medication delivery bundled with other discharge interventions reduces readmission.

Very early readmissions may be reduced with isolated bedside delivery of medications.

\section{What this study adds}

An isolated bedside medication intervention without the use of other care management efforts does not significantly affect 30-day readmission rates with a risk ratio of 1.21 (95\% CI=0.96-1.5).

Uninsured patients might benefit from a bedside intervention more than patients with commercial or private insurance.

The response to bedside delivery is likely to vary across hospitals.

$\mathrm{H}$ ospitals nationwide are evaluated on diagnosis-specific readmission rates among their Medicare beneficiaries, as part of the federal Medicare Hospital Readmissions Reduction Program that was first implemented in October 2012. ${ }^{1}$ Hospitals in Maryland have a goal of reducing readmissions, but are mandated to meet different incentives and requirements. At the start of 2014, the state of Maryland entered into a demonstration contract with the Center for Medicare and Medicaid Innovation. ${ }^{2}$ This contract requires a statewide reduction in the readmission of patients with fee-for-service Medicare discharged with any diagnosis. This reduction must match or improve upon the national Medicare readmission rate over the 5-year contract term. In practice, the Maryland Readmissions Reduction Incentive Program financially incentivizes hospitals to reduce readmission rates for patients insured by all payers as well as those who are uninsured. Like the federal program, the Maryland program accounts for disease severity and diagnosis to risk-adjust a hospital's readmission rates according to patient mix.

With the goal of statewide reduction in hospital readmissions, interventions have been implemented in Maryland to increase the likelihood that patients will thrive after discharge, including care-coordination programs. ${ }^{3}$ In 1 such intervention delivered by a large commercial pharmacy, patients received their medications at their bedside before discharge, with the goal of reducing the risk of primary nonadherence to medications prescribed during the hospitalization. It was pilot tested at 2 hospitals in the southeastern United States in 2011 with favorable results. ${ }^{4}$

Other states have implemented pharmacist-led interventions aimed at improving medication use after discharge. ${ }^{5-9}$ Hansen et al. (2011) reviewed randomized trials of bedside medication 
delivery and concluded that, in isolation, this intervention was seldom effective. ${ }^{5}$ A large, observational study at Cleveland Clinic found similar results, that is, little effect from isolated bedside delivery. ${ }^{6}$ However, preliminary results from an intervention at Barnes-Jewish Hospital in St. Louis suggested early success with delivering medications to the bedside. ${ }^{8}$

Given the promising results from the pilot study and the unique payment environment of Maryland, we hypothesized that the intervention in Maryland would reduce the risk of 30-day readmission for the patients who received bedside medication delivery relative to the risk among comparable patients who did not receive bedside medication delivery.

\section{Methods}

\section{Design}

This was a retrospective cohort study including a matched comparison group. The option for bedside delivery of medications was considered to be the standard of care in the participating hospitals and not an experimental intervention. We refer to the patients as "intervention patients" for brevity. These are the patients who received bedside medication delivery.

\section{Bedside Medication Delivery Program}

This program aimed to reduce primary medication nonadherence by expanding the role of the outpatient pharmacy. The key program component was bedside delivery of patients' medicines before discharge.

Although our analysis focuses only on inpatients, the program included not only those with inpatient hospitalizations, but also those with observation stays, emergency department visits, and outpatient surgeries. For participating patients, prescriptions for all medications that were on the discharge paperwork were sent to the on-site pharmacy. The pharmacist filled all of the requested medications unless the patient indicated that he/she did not need a refill or if the patient's insurer declined coverage. Medications were delivered directly to the patient's room by a pharmacy technician or pharmacist from the pharmacy. The pharmacy aimed to deliver the medications to the patient or caregiver within 1 to 3 hours of the scheduled hospital discharge. If a patient had questions, the technician connected the patient and pharmacist via phone or the pharmacist would be invited to the room. The pharmacy staff processed insurance verifications and approvals and collected copayments from the patient, just as if the patient had arrived at a community pharmacy.

This program was offered at 14 acute care hospitals in Maryland in 2017, which is approximately one third of all hospitals in the state. This excluded specialty hospitals. These hospitals all had the sponsoring commercial pharmacy on site. We received claims data from the Maryland Health Information Exchange for evaluation of the program from the
10 participating hospitals willing to share data for the purpose of evaluation. This project was considered exempt from review by our institutional review board.

\section{Data}

For this evaluation, we first matched the participating patients to their hospitalization data. Maryland's Health Information Exchange, called the Chesapeake Regional Information System for our Patients (CRISP), assigns unique patient identification numbers to patients hospitalized in Maryland, West Virginia, and the District of Columbia and maintains this master patient index for all patients. For this evaluation, the pharmacy sponsor securely delivered unique patient identifiers to CRISP for all of the patients who received the intervention: name, birthdate, hospital, and the date of admission and discharge that included the intervention. CRISP matched each individual receiving the intervention to his/her unique CRISP enterprise identifier number. This unique number was then used to link the patient to his/her claims as available in the Maryland case mix data maintained by the Maryland Health Services Cost Review Commission. The case mix is the all-payer claims data for the state of Maryland, which includes claims from all hospitalbased encounters, including hospital outpatient visits.

The following hierarchy was applied to search the case mix claims for data relevant to each intervention participant in order to match the intervention to the correct episode of care: (1) inpatient claims were searched for an inpatient admission at the hospital and date of discharge indicated in the source file from CRISP; (2) if no claim was found for an inpatient admission, observation stay claims were searched to see if the patient's hospitalization was coded as an observation stay; (3) if no claim was found among the observation stays, the outpatient claims were searched to see if there was an outpatient surgical or emergency room visit. A secondary approach was applied that considered variations in the date of service. The same hierarchy as above was applied, but date of service was permissible within 3 days of the intervention date in the CRISP data. This allowed for variation in billing dates or unexpected changes in patient discharge plans.

\section{Selected Comparison Group}

Next, a set of comparison patients was selected from the case mix data to loosely match the intervention patients described above. Patient matches were based on the following demographic and use criteria provided by CRISP: age group by 5-year increments, gender, hospital, and clinical characteristics of the visit. The clinical characteristics that were matched on were (a) All Patient Refined Diagnosis-Related Group (APRDRG) for the admitted patients (inpatient and observation stay patients), (b) the Current Procedural Terminology code with the highest relative value weight for the outpatient surgical 


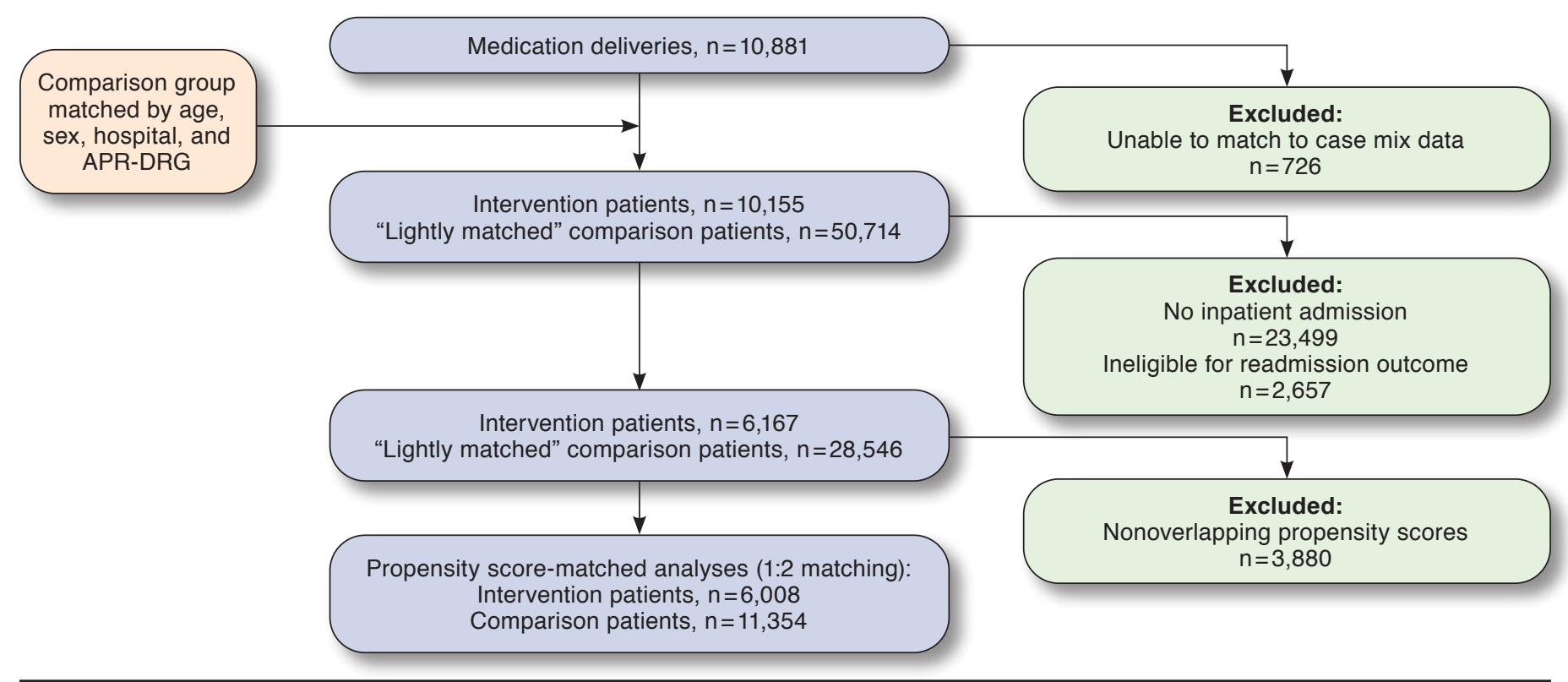

APR-DRG = All Patient Refined Diagnosis-Related Group.

patients, and (c) the Clinical Classification Software for the primary diagnosis for the emergency department-treated patients. We aimed to match 5 comparison patients for each intervention group patient.

\section{Analytic Cohort}

To create the analytic cohort, we then restricted all analyses to include only patients with an inpatient admission. Thus, we excluded patients from the intervention and comparison groups who had been hospitalized for an observational stay, since admissions after observational stays are not included in readmission metrics. We also excluded patients who were coded as ineligible for the readmission outcome because of a planned readmission scheduled in the next 30 days, and those who died during the index hospitalization.

\section{Analysis}

All analyses aimed to estimate the relative risk of 30-day readmission comparing intervention patients to a comparison group of patients. We used Poisson regression with a robust error variance and clustered by hospital for our main analyses. We began with a crude analysis comparing the 30-day readmission rates for intervention patients and the patients in the comparison group described above, and then adjusted for key covariates thought to be possible confounders of the relationship between receipt of the intervention and readmission. These covariates were gender, race, age ( $\geq 65$ years or $<65$ years), marital status, primary payer, illness severity (APR-DRG severity), mortality risk (APR-DRG mortality risk), length of stay, total charges, source of admission, and discharge disposition.

We also identified matched comparison patients by generating propensity scores to represent the probability of having been exposed to the intervention based on covariates associated with the hospitalization. The covariates included in the logistic regression model were gender, age, race, ethnicity, marital status, primary payer, admission type, major service of admission, source of admission, discharge disposition, APRDRG severity, APR-DRG mortality risk, hospital length of stay, total charges, and presence of any of the 20 most frequent diagnosis codes and 20 of the most frequent APR-DRG codes. ${ }^{10}$ We examined the distribution of the scores in the intervention and comparison groups and removed the nonoverlapping individuals from the set.

With the propensity score, we matched 1 intervention patient to 2 comparison patients using nearest neighbor matching with a caliper distance of 0.05 . We generated standardized mean differences to examine the likeness of the matched groups on potential confounders. We then estimated the relative risk of 30-day admissions comparing the intervention group and the propensity score-matched comparison group, as above, with robust Poisson regression, clustered by hospital.

We also explored inverse-probability weighting using the intervention patients and the lightly matched comparison patients to create synthetic populations. We excluded 


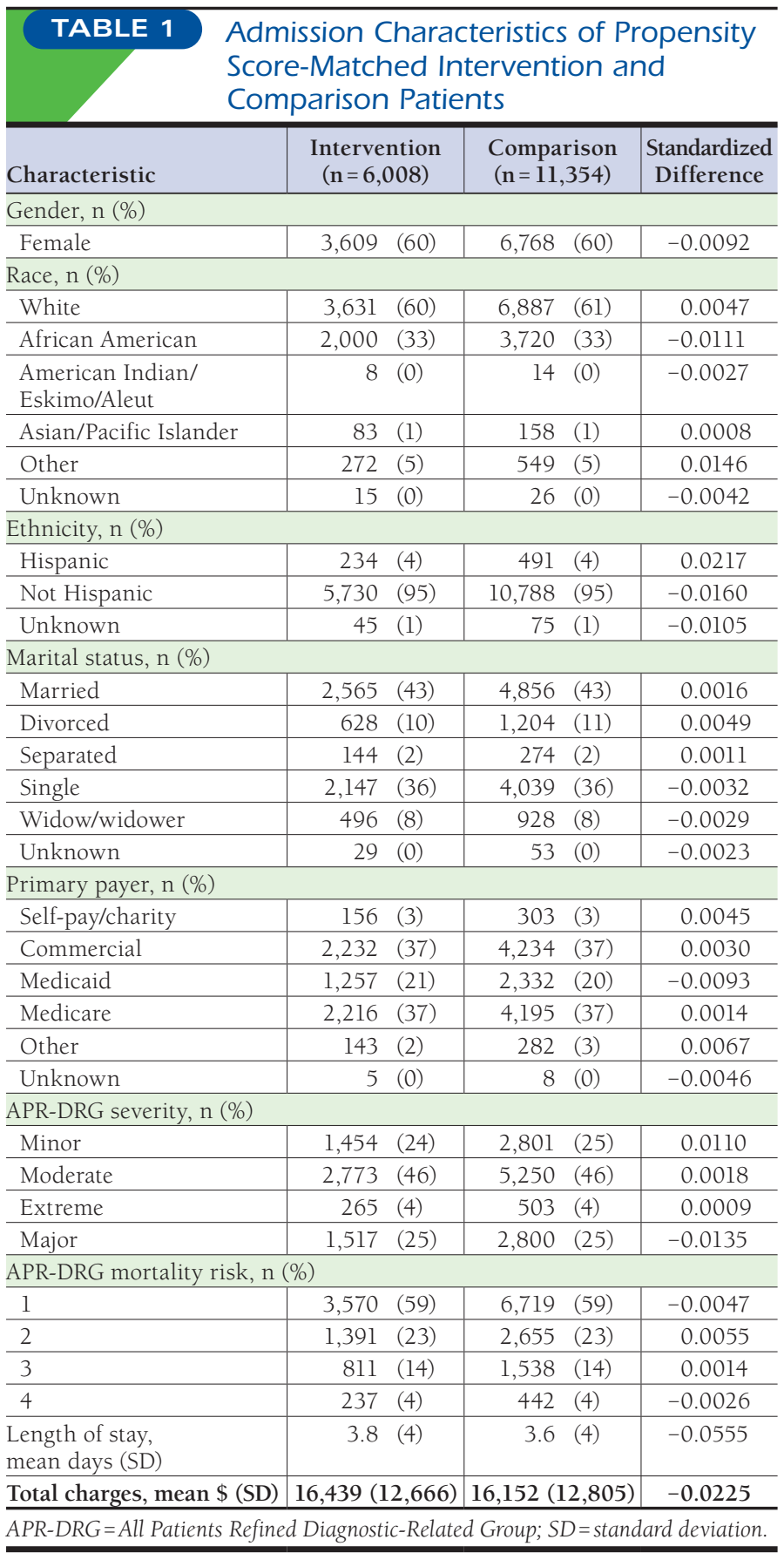

individuals with weights exceeding the 99th percentile, and repeated the Poisson regression to estimate the average treatment effect for the treated. We generated the E-value to express the required strength of an unmeasured confounder that would be required to nullify the results for our most extreme risk ratio. ${ }^{11,12}$
TABLE 2 Risk of 30-Day Readmission Associated with Bedside Medication Delivery

\begin{tabular}{|c|c|}
\hline Adjustment Method & Risk Ratio $(95 \% \mathrm{CI})$ \\
\hline Unadjusted (lightly matched) & $1.27 \quad(1.02-1.58)$ \\
\hline Adjusted $^{\mathrm{a}}$ & $1.10 \quad(1.01-1.20)$ \\
\hline Propensity score matched & $1.21 \quad(0.96-1.52)$ \\
\hline Inverse probability weighted (ATT) & $1.19 \quad(0.98-1.45)$ \\
\hline
\end{tabular}

aAdjusted for gender, race, age ( $\geq 65$ or $<65$ years), marital status, primary payer, illness severity (as APR-DRG severity), mortality risk (as APR-DRG risk), length of stay, total charges, source of admission, discharge disposition; all analyses include clustering by hospital.

APR-DRG = All Patients Refined Diagnosis-Related Groups; ATT = average treatment effect on the treated; $C I=$ confidence interval.

As sensitivity analyses, relative risk estimates were also generated using a log-binomial model in both the propensitymatched and inverse-probability weighted samples. We further explored the results in subgroups, having no prespecified hypotheses about these subgroups. We repeated the regression models with inclusion of interaction terms for the key subgroups of gender, age ( $\geq 65$ years or $<65$ years), APR-DRG mortality risk in 4 quartiles, APR-DRG severity in 4 quartiles, major diagnostic category, marital status, and primary insurer.

\section{Results}

The cohort included 6,167 patients who received the medication to bedside service while they were inpatients and 28,546 who did not from the 10 Maryland hospitals with available data (Figure 1). The 3 most prevalent major diagnostic categories, in order, were disorders of the musculoskeletal system, disorders of the respiratory system, and disorders of the circulatory system. The first reflects the many patients hospitalized for total knee and hip replacement and for spine surgery. The population was $60 \%$ female, $61 \%$ white, $31 \%$ African American, and $4.5 \%$ of patients reported as being of Hispanic ethnicity. Age was available from CRISP in age categories; the average age was around 56 years, with 34\% between ages 55 and 69 years. A total of $45 \%$ of patients were married; $32 \%$ were single; $10 \%$ were widowed; and 12\% were divorced or separated. A total of $45 \%$ had Medicare as their primary insurance; $18 \%$ had Medicaid; and 33\% had commercial insurance. The average length of stay of the index admission was 3.8 days.

Before propensity score matching, the lightly matched groups were fairly similar (Appendix A, available in online article). After propensity score matching, the intervention and comparison groups were well matched. Less than $1 \%$ of the total sample was removed with trimming the nonoverlapping populations. The standardized mean differences were very close to zero for all evaluated covariates (Table 1).

In this propensity score-matched set of patients, the relative risk of 30-day readmission from the robust Poisson regression model, which included no covariates except treatment group 


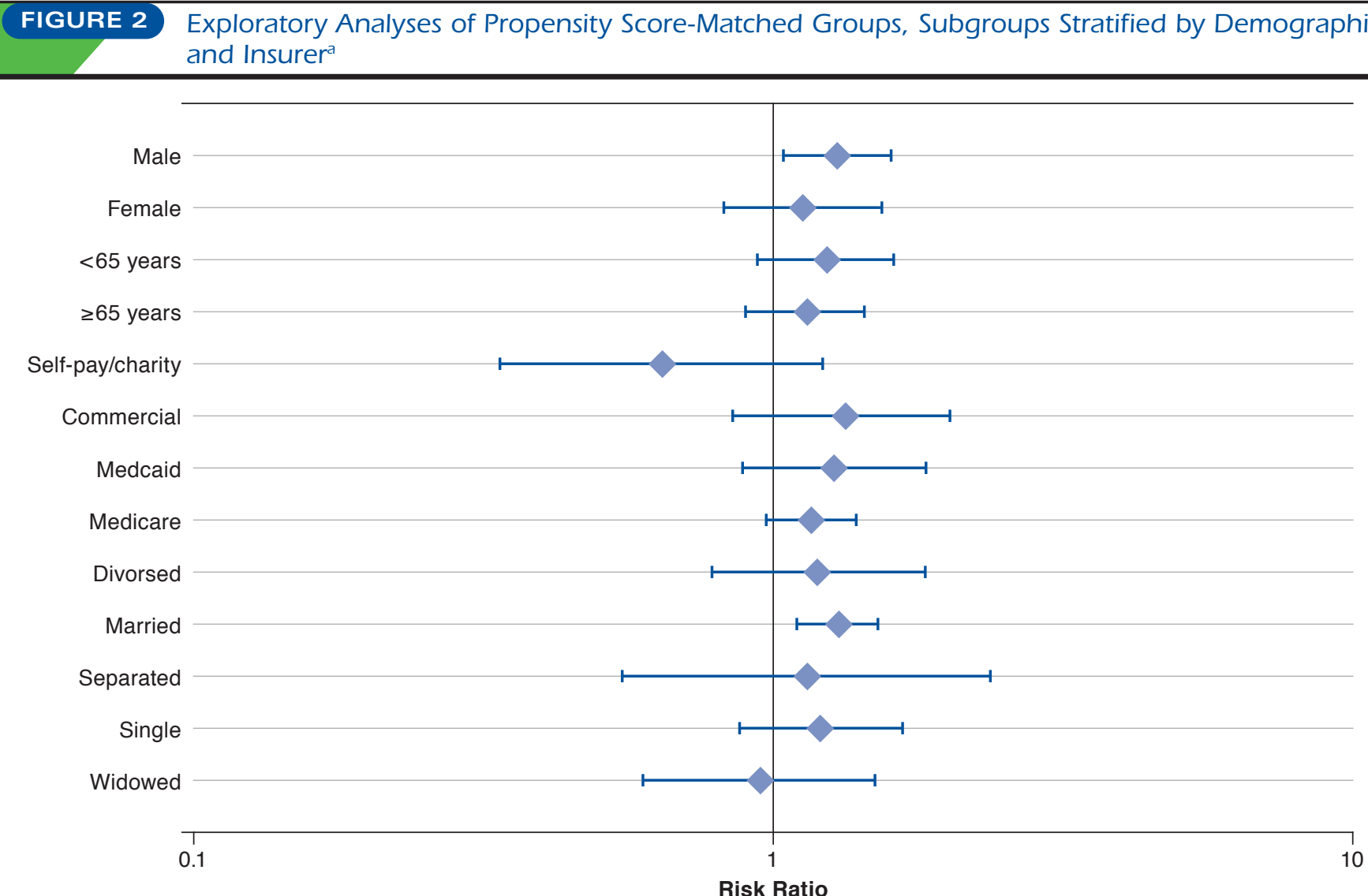

${ }^{a}$ See Appendix B for results of interaction term testing.

and was clustered by hospital, was 1.21 (95\% CI=0.96-1.52), suggesting a higher readmission risk among patients receiving the intervention, although this was not statistically significant (Table 2). The inverse-probability weighted model yielded similar results with a risk ratio of 1.19 (95\% CI=0.98-1.45).

There were 2 subgroups that appeared to be harmed from the intervention (men and married patients) in stratified analyses, but the models that included interaction terms did not suggest that this was a statistically significant finding. There was a significant interaction of the intervention by payer with uninsured patients potentially benefitting from the intervention more than those with commercial or private payers. There was a significant interaction between the intervention and some of the individual hospitals, suggesting possibly heterogeneous effects of the intervention by hospital, but in no hospital was the treatment effect significantly different from 1.0 in stratified analyses. As described in the methods, in the main models we clustered the standard errors by hospital (Figure 2 and Appendix B, available in online article).
The E-value calculation revealed that the observed risk ratio of 1.21 in the propensity-matched model could be explained away by an unmeasured confounder that was associated with both the treatment and the outcome by a risk ratio of 1.7 , but weaker confounding could not do so.

\section{Discussion}

We did not find an effect on 30-day readmission rates from the isolated bedside delivery of medications to inpatients. Although the risk ratio attributable to bedside delivery exceeded 1.0 consistently across all analyses, the confidence interval included 1.0 for most analyses.

Several other interventions have attempted to reduce 30-day readmissions with isolated bedside delivery of medications. Interventions that had been tested in randomized controlled studies were reviewed by Hansen et al. who concluded that, in isolation, this intervention was seldom effective..$^{5}$ They observed that bundled interventions, which included postdischarge phone calls, were more likely to be effective. A large observational study at Cleveland Clinic found similar 
results, that is, little effect from isolated bedside delivery. ${ }^{6}$ After adjusting for baseline differences in patients who did and did not have the intervention, the bedside delivery of medicines was not an independent predictor of 30-day readmissions. However, they found the intervention to have a statistically significant effect on readmission within 14 days. These authors concluded that because patients opted into receiving medications, the study results may have been affected by a healthy user bias, where patients who chose to receive medicines may also have been more likely to engage in other interventions that lowered their risk of readmissions. They cautioned that the readmission occurrences were only observable within their own health system, but they thought that this was unlikely to bias the results. ${ }^{7}$

Preliminary results from an intervention at Barnes-Jewish Hospital suggested early success in delivering medications to the bedside but did not report on outcomes of delivery. ${ }^{8}$ Johns Hopkins Hospital implemented a bedside delivery service coordinated by a transitions pharmacist extender aimed at lessening primary nonadherence. This was 1 element of a broader effort to implement transitions-of-care services across the hospital. This intervention did not specifically target 30-day readmission reductions and did not report on this outcome. ${ }^{9}$

Might the intervention have been harmful? It is worth thinking through mechanisms by which harm might be done by isolated bedside delivery. Although the pharmacy would not have filled the medication if the insurer reported that it was an early fill, we still think it is possible that patients may have gone home with medications that duplicated those at home, leading to confusion and medication error. Alternatively, some patients may have taken home medicines that were meant to replace home medicines and experienced difficulty reconciling their new and existing medications without a follow-up phone call after discharge. While this challenge might also occur among patients seeking to fill discharge prescriptions at their usual pharmacies, confusion might be greater among those filling prescriptions at the hospital based on difficulty reviewing their home medicines before the fill. Additionally, the prompt fill of medicines at the time of discharge may not allow patients to review medication changes with their primary care doctors or other key clinicians.

Finally, there may be fewer safeguards against inappropriate polypharmacy (such as taking 2 drugs from the same class) when multiple pharmacies are used to fill medications, particularly if there is suboptimal reconciliation of medications between pharmacies. We know of no research that has rigorously explored these possibilities.

\section{Limitations}

We recognize that in this nonrandomized intervention there may be channeling bias if the intervention was directed at patients thought to be at greater risk of readmission. Thus, the patients receiving the intervention may have been at higher risk of readmission, even after controlling for many known factors associated with readmission. However, it is unlikely that there are unmeasured confounders sufficient that adjustment would make the point estimate and its confidence interval move to below 1.0 (suggesting a statistically significant benefit of the intervention).

Our adjustment techniques, including propensity score matching and inverse-probability weighting, suggested that the treated and comparison patients were very similar on the measured potential confounders. Certainly, there is the possibility of residual confounding by unmeasured factors that made the treated patients at greater risk of readmission, such as psychosocial determinants of health that are not well captured in claims data. However, in the exploratory analyses that looked at the effect of the intervention by payer, patients with Medicaid as their insurer had a similar risk ratio as patients with commercial insurance or with Medicare. Patients without the support of a spouse had a similar risk ratio as the married patients, suggesting that these patients similarly experienced little benefit from the bedside delivery. We note that the comparison patients may not have been prescribed medications at discharge, as this could not be known from the data. This, too, might have made the comparison patients somewhat healthier than the intervention patients.

Potential benefit might have been obscured by heterogeneity in how the intervention was delivered, as this involved multiple hospitals with diverse staff. We do not have knowledge of the patients who were offered the intervention and declined, nor do we have a record of the patients who were never approached. There may have been systematic differences in the selection of patients across hospitals or in how the intervention was delivered across hospitals, as suggested by a significant hospital-intervention interaction. When we explored the major diagnostic categories to see if there was a subset of admissions where patients benefited from this intervention, patients with metabolic or endocrine disorders disproportionately benefited from bedside delivery of medications. It may be worth exploring further to see if accessing insulin is a particular challenge to patients at discharge, but this was not a prespecified hypothesis.

\section{Conclusions}

Isolated bedside delivery of medications does not affect 30-day readmissions. We expect, however, that it may have benefited patients in ways that were not captured in this readmission metric. Bedside delivery necessarily reduces primary adherence, which may improve patient-valued outcomes other than 30-day readmission rates. We expect that bedside delivery as an isolated intervention is insufficient to affect 30-day readmissions, but might prove to be an important part of a bundle of transition services that enable patients to thrive after discharge. 


\section{Authors}

JODI B. SEGAL, MD, MPH; ARIELLA APFEL, MHS; DANIEL J BROTMAN, MD; and JEANNE M. CLARK, MD, MPH, Division of General Internal Medicine, and KENNETH M. SHERMOCK, PharmD, PhD, Division of General Internal Medicine and Health System Department of Pharmacy, The Johns Hopkins University School of Medicine, Baltimore, Maryland.

AUTHOR CORRESPONDENCE: Jodi B. Segal, MD, MPH, 624 N. Broadway, Rm. 644, Baltimore, MD 21205.

Tel.: 410.955.9866; E-mail: jsegal@jhmi.edu.

\section{DISCLOSURES}

This study was funded by Walgreen Co. through unrestricted funds to Johns Hopkins University, which has received fees from Walgreens for providing consultation as an institution to Walgreens. This arrangement has been reviewed and approved by the Johns Hopkins University in accordance with its conflict of interest policies. Segal received a grant from the National Institute on Aging during the conduct of this study. The other authors have nothing to disclose.

\section{ACKNOWLEDGMENTS}

The authors acknowledge Ross D. Martin, MD, MHA, FAMIA, Program Director, CRISP Research Initiative; Audrey Speter, Director of Healthcare Policy, hMetrix; and Jineesh K. James, Senior Vice President, hMetrix, for their contributions to this study.

\section{REFERENCES}

1. Centers for Medicare and Medicaid Services. Baltimore, MD. Hospital Readmissions Reduction Program (HRRP). Available at: https://www.cms. gov/medicare/medicare-fee-for-service-payment/acuteinpatientpps/readmissions-reduction-program.html. Updated April 15, 2019. Accessed February 7, 2020.
2. Maryland Hospital Services Cost Review Commission. Readmission Reduction Incentive Program (RRIP). Available at: https://hscrc.state.md.us/ Pages/init-readm-rip.aspx. Accessed February 7, 2020.

3. Hoyer EH, Brotman DJ, Apfel A, et al. Improving outcomes after hospitalization: a prospective observational multicenter evaluation of care coordination strategies for reducing 30-day readmissions to Maryland hospitals. J Gen Intern Med. 2018;33(5):621-27.

4. Kirkham HS, Clark BL, Paynter J, Lewis GH, Duncan I. The effect of a collaborative pharmacist-hospital care transition program on the likelihood of 30-day readmission. Am J Health-Syst Ph. 2014;71(9):739-45.

5. Hansen LO, Young RS, Hinami K, Leung A, Williams MV. Interventions to reduce 30-day rehospitalization: a systematic review. Ann Intern Med. 2011;155(8):520-28.

6. Lam SW, Sokn E. Effect of pharmacy-driven bedside discharge medication delivery program on day 30 hospital readmission. J Pharm Pract. 2019:897190019825961.

7. Baker M, Bell CM, Xiong W, et al. Do combined pharmacist and prescriber efforts on medication reconciliation reduce postdischarge patient emergency department visits and hospital readmissions? J Hosp Med. 2018;13(3):152-57.

8. Thompson CA. Pharmacy departments innovate to reduce readmissions penalty. Am J Health Syst Pharm. 2013;70(4):296-98.

9. Gilmore V, Efird L, Fu D, LeBlanc Y, Nesbit T, Swarthout M. Implementation of transitions-of-care services through acute care and outpatient pharmacy collaboration. Am J Health Syst Pharm. 2015;72(9):737-44

10. Averill RF, Goldfield N, Hughes JS, et al; 3M Health Information Systems, National Association of Children's Hospital and Related Institutions, Medical Advisory Committee for NACHRI APR-DRG Research Project. All Patient Refined Diagnosis Related Groups (APR-DRGs): methodology overview. Version 20.0. July 2003. Available at: https://www.hcup-us. ahrq.gov/db/nation/nis/APR-DRGsV20MethodologyOverviewandBibliograp hy.pdf. Accessed February 7, 2020.

11. VanderWeele TJ, Ding P. Sensitivity analysis in observational research: introducing the E-value. Ann Intern Med. 2017;167(4):268-74.

12. VanderWeele TJ, Mathur MB, Ding P. Correcting misinterpretations of the E-value. Ann Intern Med. 2019;170(2):131-32. 


\begin{tabular}{|c|c|c|c|}
\hline APPENDIX A & $\begin{array}{l}\text { Baseline Cha } \\
\text { Intervention } \\
\text { Comparison }\end{array}$ & $\begin{array}{l}\text { aracteristics o } \\
\text { and Lightly } \\
\text { Patients }\end{array}$ & Matched \\
\hline Characteristics & $\begin{array}{l}\text { Exposure } \\
(\mathrm{n}=6,167)\end{array}$ & $\begin{array}{c}\text { Control } \\
(\mathrm{n}=28,546)\end{array}$ & $\begin{array}{c}\text { Standardized } \\
\text { Difference }\end{array}$ \\
\hline Gender, n (\%) & & & \\
\hline Female & $3,687(60)$ & $17,015(60)$ & -0.0041 \\
\hline Race, n (\%) & & & \\
\hline White & $2,051 \quad(33)$ & $8,869(31)$ & -0.0471 \\
\hline African American & $8(0)$ & $50(0)$ & 0.0258 \\
\hline $\begin{array}{l}\text { American Indian/ } \\
\text { Eskimo/Aleut }\end{array}$ & $89(1)$ & $636(2)$ & 0.0602 \\
\hline Asian/Pacific Islander & $281(5)$ & $1,489(5)$ & 0.0278 \\
\hline Other & $21(0)$ & $117(0)$ & 0.0169 \\
\hline Unknown & $3,717(60)$ & $17,385(61)$ & 0.0123 \\
\hline Ethnicity, n (\%) & & & \\
\hline Hispanic & $236(4)$ & $1,315(5)$ & 0.0399 \\
\hline Not Hispanic & $5,862(95)$ & $26,834(94)$ & -0.0455 \\
\hline Unknown & $45(1)$ & $292(1)$ & 0.0327 \\
\hline Marital status, n (\%) & & & \\
\hline Married & $636(10)$ & $2,685(9)$ & -0.0302 \\
\hline Divorced & $2,619(42)$ & $13,091(46)$ & 0.0685 \\
\hline Separated & $149(2)$ & $561(2)$ & -0.0273 \\
\hline Single & $2,218(36)$ & $8,923(31)$ & -0.0996 \\
\hline Widow/widower & $31(1)$ & $240(1)$ & 0.0373 \\
\hline Unknown & $514(8)$ & $3,046(11)$ & 0.0819 \\
\hline Primary payer, $\mathrm{n}(\%)$ & & & \\
\hline Self-pay/charity & $157(3)$ & $1,102(4)$ & 0.0734 \\
\hline Commercial & $2,265(37)$ & $9,972(35)$ & -0.0376 \\
\hline Medicaid & $1,307(21)$ & $5,090(18)$ & -0.0859 \\
\hline Medicare & $2,283(37)$ & $11,786(41)$ & 0.0882 \\
\hline Other & $150(2)$ & $581(2)$ & -0.0273 \\
\hline APR-DRG severity, n (\%) & & & \\
\hline Minor & $2,851(46)$ & $12,305(43)$ & -0.0624 \\
\hline Moderate & $1,472(24)$ & $7,457(26)$ & 0.0508 \\
\hline Extreme & $286(5)$ & $1,533(5)$ & 0.0367 \\
\hline Major & $1,558(25)$ & $7,251(25)$ & 0.0023 \\
\hline APR-DRG mortality risk & $\mathrm{n}(\%)$ & & \\
\hline 1 & $3,652(59)$ & $16,028(56)$ & -0.0608 \\
\hline 2 & $1,426(23)$ & $6,515(23)$ & -0.0071 \\
\hline 3 & $835(13)$ & $4,745(17)$ & 0.0868 \\
\hline 4 & $254(4)$ & $1,258(4)$ & 0.0149 \\
\hline $\begin{array}{l}\text { Length of stay, } \\
\text { mean days (SD) }\end{array}$ & $3.8(4)$ & $3.8(4)$ & 0.0035 \\
\hline $\begin{array}{l}\text { Total charges, } \\
\text { mean \$ (SD) }\end{array}$ & $16,513(12,799)$ & $15,611(14,562)$ & -6.5818 \\
\hline
\end{tabular}




\section{APPENDIX B Exploratory Subgroup Analyses ${ }^{\mathrm{a}}$}

\begin{tabular}{|c|c|c|}
\hline & $\begin{array}{c}\text { Relative Risk } \\
\text { of Readmission } \\
\text { (Exposure vs. } \\
\text { Control) }\end{array}$ & $95 \% \mathrm{CI}$ \\
\hline \multicolumn{3}{|l|}{ Gender } \\
\hline Male & 1.30 & $(1.05-1.60)$ \\
\hline Female & 1.13 & $(0.83-1.54)$ \\
\hline \multicolumn{3}{|l|}{ Age, years } \\
\hline$<65$ & 1.24 & $(0.95-1.61)$ \\
\hline$\geq 65$ & 1.14 & $(0.91-1.44)$ \\
\hline \multicolumn{3}{|l|}{ By insurance } \\
\hline Self-pay/charity & 0.65 & $(0.34-1.22)^{b}$ \\
\hline Commercial & 1.34 & $(0.86-2.02)$ \\
\hline Medicaid & 1.28 & $(0.89-1.83)$ \\
\hline Medicare & 1.17 & $(0.98-1.39)$ \\
\hline Other/unknown & 0.16 & $(0.04-0.62)^{b}$ \\
\hline \multicolumn{3}{|l|}{ By marital status } \\
\hline Divorced & 1.20 & $(0.79-1.84)$ \\
\hline Married & 1.30 & $(1.11-1.52)$ \\
\hline Separated & 1.14 & $(0.55-2.38)$ \\
\hline Single & 1.21 & $(0.88-1.67)$ \\
\hline Unknown & 0.66 & $(0.27-1.61)$ \\
\hline Widowed & 0.95 & $(0.60-1.50)$ \\
\hline \multicolumn{3}{|c|}{ By hospitals (with more than 600 exposed inpatients) } \\
\hline A & 1.77 & $(1.33-2.34)$ \\
\hline $\mathrm{B}$ & 1.55 & $(1.23-1.94)$ \\
\hline C & 0.76 & $(0.53-1.09)^{\mathrm{b}}$ \\
\hline $\mathrm{D}$ & 0.69 & $(0.42-1.12)^{b}$ \\
\hline \multicolumn{3}{|l|}{ By MDC } \\
\hline 4 (respiratory system) & 1.27 & $(1.11-1.44)$ \\
\hline 5 (circulatory system) & 1.24 & $(0.91-1.68)$ \\
\hline 6 (digestive system) & 1.38 & $(1.12-1.71)$ \\
\hline 8 (musculoskeletal system) & 1.53 & $(1.19-1.96)$ \\
\hline 10 (endocrine, metabolic system) & 0.60 & $(0.43-0.82)^{b}$ \\
\hline 14 (pregnancy) & 0.55 & $(0.17-1.78)$ \\
\hline \multicolumn{3}{|l|}{ APR-DRG mortality risk } \\
\hline 1 & 1.14 & $(0.81-1.61)$ \\
\hline 2 & 1.50 & $(1.17-1.91)$ \\
\hline 3 & 0.94 & $(0.72-1.23)$ \\
\hline 4 & 1.23 & $(0.94-2.35)$ \\
\hline \multicolumn{3}{|l|}{ APR-DRG severity } \\
\hline Minor & 0.98 & $(0.41-1.60)$ \\
\hline Moderate & 1.22 & $(0.93-1.60)$ \\
\hline Major & 1.14 & $(0.98-1.34)$ \\
\hline Extreme & 1.38 & $(0.99-1.93)$ \\
\hline
\end{tabular}

aRelative risks of readmission stratified by subgroups using robust Poisson regression with clustering by hospital in the propensity score-matched cohort. In separate models, each subgroup was evaluated with inclusion of an interaction term, interacting the intervention and the subgroup variable of interest.

${ }^{b}$ Significant interaction term.

$A P R-D R G=$ All Patients Refined Diagnostic-Related Group; $C I=$ confidence interval; $M D C=$ major diagnostic category. 